\title{
NEW OBSERVATIONS OF BL LACERTAE OBJECTS
}

\author{
JOHN T. STOCKE \\ Center for Astrophysics and Space Astronomy \\ University of Colorado - Boulder \\ Boulder, CO 80309-0389 USA
}

\begin{abstract}
This contribution is divided into three parts: 1. A summary of mostly published work that describes the new type of BL Lac Objects discovered with X-ray satellites, "X-ray Bright BL Lacs (XBLs)", and their relationship to the previously known "radio bright BL Lacs (RBLs)." Most (but not all?) of the differences between XBLs and RBLs are explicable using a "viewing angle" model in which the soft X-ray emission emanates into a much wider cone $\left(23-30^{\circ}\right)$ than the radio emission $\left(8-10^{\circ}\right)$. 2. New ROSAT PSPC soft X-ray observations are presented for complete samples of XBLs and RBLs which may explain the absence of luminous optical emission lines in BL Lacs. 3. A deep, subarcsec optical imaging survey of a large (50) sample of both XBLs and RBLs conducted at the CanadaFrance-Hawaii $3.6 \mathrm{~m}$ Telescope (CFHT), whose purpose was to characterize the host galaxies and clustering environment of BL Lacs. This work defines more precisely the "parent population" of BL Lacs and identifies a small number of discrepant objects which may not be "beamed FR $1 \mathrm{~s}$ ".
\end{abstract}

\section{X-Ray Loud BL Lacertae Objects (XBLs)}

The discovery of numerous new BL Lac Objects with various X-ray satellite telescopes (Einstein, EXOSAT, ROSAT) has revitalized BL Lac research by giving us many new objects to study, new models to create and new disagreements to discuss. With $\sim 200 \mathrm{BL}$ Lacs now known, it is evident that, while XBLs and RBLs share enough common features to both be called BL Lacs, these groups differ systematically in the following ways:

RADIO: While most XBLs and RBLs have the extended radio power levels and morphologies of FR $1 \mathrm{~s}$, their core dominance values differ sys- 
tematically as would be expected if we are viewing them from different angles relative to their jet axes. Specifically, using data from Perlman \& Stocke (1993) as updated by Rector et al. (1996) to constitute nearly complete samples of RBLs and XBLs we find the mean core dominance values for FR 1s:XBLs:RBLs to be 0.1:1.5:20 suggesting (for a single assumed $\Gamma=5$ ) mean viewing angles of $60^{\circ}: 23^{\circ}: 10^{\circ}$ and relative volume densities of 130:5:1. These values are similar to those suggested by Urry \& Padovani (1995) from other considerations. However, the RBL sample contains a larger number (although still a small percentage) of high-z, high radio power level sources which appear to be FR 2s rather than FR 1s.

OPTICAL: While the optical spectra of both RBLs and XBLs can both be described as nearly featureless (Stickel et al. 1991; Morris et al. 1991), many more RBLs are either very featureless or possess only weak, low luminosity emission lines (Rector et al. 1996). High quality optical spectra of XBLs almost always show weak absorption features of of an underlying host galaxy. The maximum optical polarization percentages of the class of XBLs are significantly less than RBLs and, most interestingly, $~ 80 \%$ of XBLs show "preferred position angles" $\left( \pm 10^{\circ}\right)$ of optical polarization, which RBLs rarely show (Jannuzi et al. 1994). The preferred polarization position angles seem particularly suggestive of viewing angle differences.

X-RAY: The soft (0.1-2.4 keV) X-ray spectra of RBLs and XBLs are quite similar and both have significantly steeper spectra than quasars $\left(\langle\alpha\rangle_{p h}\right.$ $=-2.2$ and -2.4 for RBLs and XBLs compared to -1.5 for quasars; Perlman et al. 1996; Urry et al. 1996). At harder X-ray energies the spectra of RBLs may flatten while XBLs steepen (Sambruna et al. 1994). The $\gamma$-ray detections of BL Lacs are dominated by RBLs (von Montigny et al. 1995).

These various properties are explicable if XBLs are viewed further from the jet axis than RBLs, requiring the soft X-ray beam to be broader (but not necessarily at lower $\Gamma$ ) than the radio and optical beams. However, the overall spectral energy distributions (SED) of XBLs and RBLs differ significantly, with the XBL SED peaking in the UVX while the RBL SED peaks in the IR/optical. Such a large difference is difficult to understand in the context of the simplest viewing angle models and may require other differences between XBLs and RBLs (e.g. electron energy distribution; Sambruna et al. 1996; Padovani \& Giommi 1995). The large difference in $\left\langle V / V_{\max }\right\rangle$ values for RBLs and XBLs (0.62 and 0.34 ) also remains a problem for the unification of these objects. These new best values reflect the addition of 3 new BL Lacs to both the 1 Jy RBL and EMSS XBL samples, including three low luminosity XBLs discovered recently with the ROSAT HRI (Rector et al. 1996) as suggested by Browne \& Marcha (1993). 


\section{Soft X-Ray Spectra and the Absence of Strong Emission Lines}

The viewing angle model may also explain the absence of luminous emission lines in BL Lacs. Guilbert, Fabian \& McCray (1983; GFM83) suggested that the emission line regions in BL Lacs could be unstable due to having a very soft $\mathrm{X}$-ray emission spectrum. In contrast, the harder X-ray spectra of quasars create a quasi-stable, two-phase medium, similar to the ISM of the Galaxy, in which the warm clouds imbedded in a hot substrate are the broad (and possibly also the narrow) emission line clouds. By this model the very soft X-ray spectra of BL Lacs are insufficient to create a substrate hot enough to allow quasi-stable warm clouds. While our new ROSAT PSPC survey of BL Lacs supports this hypothesis, the hard X-rays seen in some BL Lacs (primarily RBLs) seem to make the GFM83 model untenable.

However, in the viewing angle model, the soft X-ray emission emanates from the nucleus in a significantly wider cone than the radio (and inverseCompton X-ray) emission. Thus, the potential emission line regions of $\mathrm{BL}$ Lacs are illuminated primarily by the steep, soft X-ray continuum, not by the harder inverse-Compton emission, making warm clouds unstable over much of the near-nuclear region and suppressing luminous line emission.

\section{The CFHT Deep Optical Imaging Survey of BL Lac Objects}

A deep optical imaging survey of the host galaxy and meta-galactic environment of 50 BL Lac Objects at $z<0.65$ was conducted at CFHT in Gunn-r band and typically during subarcsec seeing (WSY96; W96). These observations were sufficient to resolve 46 of the $50 \mathrm{BL}$ Lacs observed, 32 well-enough to determine an unambiguous host galaxy morphology. Of these 32,29 are definite or likely ellipticals and 3 are spirals (PKS 1413+135; MS 0205+351; OQ 530). MS $0205+351$ also has the lowest luminosity "host galaxy" in the survey $\left(M_{r}=-21.7\right.$ compared to a $\left.\left\langle M_{r}\right\rangle=-23.2\right)$ and is the only object whose core is definitely decentered $(>0.1$ arcsec) with respect to its "host" (Stocke, Wurtz \& Perlman 1995). An HST image confirms that the "host" of PKS $1413+135$ is an edge-on spiral (McHardy et al. 1994). The VLBI structure of PKS $1413+135$ is unique amongst BL Lacs in being twosided, clearly a problem for relativistic beaming models; see Perlman et al. (this conference) for a stunning VLBA map. These unusual BL Lacs are candidates for background quasars whose properties are, at least partially, produced by microlensing due to stars in a foreground galaxy.

Our analysis of BL Lac clustering environments confirms the Prestage \& Peacock (1988) result that BL Lacs avoid rich clusters. W96 finds that $<B_{g g}>(\mathrm{BL}$ Lacs $)=250 \mathrm{Mpc}^{1.77}$, where $B_{g g}$ is the amplitude of the BL Lacgalaxy covariance function. For reference Abell richness classes $0,1 \& 2$ have $\left.<B_{g g}\right\rangle=360,645,945 \mathrm{Mpc}^{1.77}$, so that a typical BL Lac environment is 
that of an Abell richness class $\leq 0$ cluster. Only five BL Lacs are in richness $\geq 1$ clusters and four of those are at $z \geq 1 / 2$ (the low-z, rich cluster BL Lac is PKS 0548-322). Despite the long-standing belief that BL Lacs are "beamed" FR 1 radio galaxies, the host galaxies and clustering environs of BL Lacs are better matched by FR 2s than by FR $1 \mathrm{~s}$ in the sense that $\sim 20 \%$ of FR $1 \mathrm{~s}$ are in brighter host galaxies and richer clusters than any BL Lac (these are rich cluster brightest cluster galaxies; BCGs). The evolution in BL Lac cluster environments is also better matched by FR $2 \mathrm{~s}$ than by FR 1s. But because the extended radio power levels and morphologies of most BL Lacs are those of FR 1s, we do NOT suggest that BL Lacs are beamed FR 2s. Rather we suggest a minor modification of the BL Lac parent population so that the FR 1 radio galaxies in the richest clusters (and specifically BCGs) cannot be BL Lacs. Following Bicknell (1994) this could be due to a more rapid deceleration of the radio jet by the enhanced ICM pressure in a rich cluster or to a greater collimation of the jet in rich cluster FR 1s compared to FR 1s in poor clusters (Stocke \& Burns 1978). This also explains the absence of BL Lacs in a large sample of Abell cluster FR 1s (Owen, Ledlow \& Keel 1996).

This report is a very brief summary of the $\mathrm{PhD}$ dissertations of Drs. Eric Perlman and Ron Wurtz and of Mr. Travis Rector at the University of Colorado. This work was supported by a NASA Long Term Space Astrophysics Grant to JTS.

\section{References}

Bicknell, G.V. $1994 A p J, 422,542$.

Browne, I.W.A. \& Marcha, M.J.M. 1993 MNRAS, 261, 795.

Guilbert, P.W., Fabian, A.C. \& McCray, R. $1983 A p J$, 266, 466 (GFM83).

Jannuzi, B.T., Smith, P. \& Elston, R. 1994 ApJ, 428, 130.

McHardy, I.M. et al. $1994 M N R A S, 268,681$.

Morris, S.L. et al. $1991 A p J, 380,49$.

Owen, F.N., Ledlow, M.J. \& Keel, W.C. 1996, $A J$, Jan. issue.

Padovani, P. \& Giommi, P. 1995 ApJ, 444, 567.

Perlman, E.S. \& Stocke, J:T. 1993 ApJ, 406, 430.

Perlman, E.S., Stocke, J.T., Wang, Q.D. \& Morris, S.L. 1996 ApJ Jan. 10 issue.

Prestage, R.M. \& Peacock, J.A. 1988 MNRAS, 230, 131.

Rector, T., Stocke, J.T. \& Perlman, E.S. 1996, in prep.

Sambruna, R.M., Urry, C.M. \& Maraschi, L. $1996 A p J$, in press.

Sambruna, R.M. et al. $1994 A p J, 434,468$.

Stickel, M., Padovani, P., Urry, C.M. \& Fried, J.W. 1991 ApJ, 374,431.

Stocke, J.T. \& Burns, J.O. 1978 ApJ, 319, 671.

Stocke, J.T., Wurtz, R. \& Perlman, E.S. 1995 ApJ, Nov 10.

Urry, C.M. \& Padovani, P. 1995 PASP, 107,803.

Urry, C.M. et al. $1996 A p J$, in press.

Wurtz, R., Stocke, J.T. \& Yee, H.K.C. 1996 ApJS, Mar issue (WSY96).

Wurtz, R., Stocke, J.T., Ellingson, E.E. \& Yee, H.K.C. 1996, in prep. (W96).

von Montigny, C. et al. 1995 ApJ, 440, 525. 